\title{
Applications of Non-Contact Digital Image Correlation Method (DIC) - A Review
}

\author{
K Sharmila Sai Sree*, Srikanth Koniki ${ }^{l}$ \\ * PG student, Civil Engineering Department, GRIET, Hyderabad, India. \\ 1 Associate Professor, Civil Engineering Department, GRIET, Hyderabad, India.
}

\begin{abstract}
The non-contact digital image of the correlation method on thermal strains has been studied to know the capability of measuring the variations in strains occurring in the alloy surface. By digital image correlation method, we can confirm the strains by heating and cooling of the alloy due to temperatures of sunlight, etc. The techniques are developed and implemented in thermal heating strains are considered through the Noncontact digital image of correlation method. The material can expand or be compressed depending on time variation, temperature changes, and strains generated. There can be a thermal shock which means a sudden change resulting in high variations so to control the results we are doing a study. There are different types of correlation methods which are a naturalistic method, survey method, and archival method in our research we are developing the noncontact analysis which can compare the strains that occurred before and after loads occurred by temperature. The results show the easiest method of analyzing different aspects.
\end{abstract}

\section{Introduction}

The process of establishment of a harm sensing device that gives strategy in engineering structures from damage is named structural health monitoring (SHM) which is considered in DIC [1]. The damage is observed by the digital image correlation method due to the changes which are threatened by environmental effects and material degradations. DIC plays a major role in detecting damage caused by external and internal changes. The damage in geometrical structures can be observed by analyzing through this method [2]. The DIC involves progression under the opinion of the system and its dynamic responses \& capacities. DIC is used for controlling and achieving the properties of materials should consider sustainability, quality, serviceability, and durability. This method can be conducted in any kind of department like oceanology, mechanical, civil, electrical, computer, and many other departments. Important things to be considered are the sustainability of structure and should be beneficial to environment and society which does not harm anyways. Correlation is a method that can compare and gives the result by distances, heights, materials, etc. According to the changes that occurred on structures it can give temperature, time, stresses, strains, deflections, frequency, amplitude, etc as the results. It also has certain boundary conditions and limitations that can affect or shows the changes in systems performance [3]. The DIC involves the process under real-time analysis and gives accurate results. In extremely worst conditions by the damage and different environmental disasters, it can reanalyze the structures by the DIC method. Since the start of the 1980's the technique is introduced and from then different investigations are improved in many aspects. The quality in digital image correlation method major modifications is done in algorithms. Especially it can detect the damage on the surface layer by imaging process by speckle method. Digital image processing has been increased in popularity mainly in microscopic and nano-scale measurements. Researchers are identifying the problems or damage that occurred at any kind of materials that could help damage control. Different problems can be solved by the DIC method:

1) Detecting the damage on structures;

2) Positioning the injury;

3) Categorizing types of damage;

4) Measuring harshness of damage.

From the below fig 1 process of DIC method is shown. 


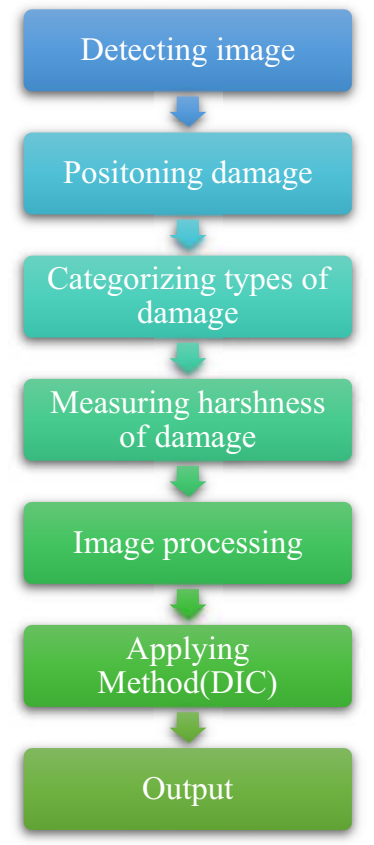

Figure 1 Process of DIC

So, to overcome these problems DIC is addressed in the context which is divided in four parts. The first part tells about the identification of damage on structures so that the next control can be done and it should be identified before the damage is occurred [1]. Then it follows data collection, stabilization, and cleansing.

\section{Applications Of Dic:}

This section presents a comprehensive review of research studies conducted on composites. Here in this literature review, there are different types of experiments conducted on the method of Digital Image Correlation (DIC) in previous years.

Study of non-contact digital image correlation method will consider all different kind of materials example: Alloys, wood, plastics, foams, signals, food packing materials, etc which changes its original to different positions.

\subsection{Study of DIC based on civil structures}

Pressure sensors for structural health monitoring are about a detailed study on matrix-created sensors which shows the acts of buildings, bridges, etc health which is threatened by earthquakes, environmental effects, and materials degradations. Here SHM is a smart method where sensors are placed and selfsensing is applied. It gives structural health of structure and details of loads, pressures which are affected by external or internal changes by this output we can control the damage before it gets affected [1]. Newton Raphson Digital Image Correlation Method on Full-Field Displacement and Strain Calculation on $2 \mathrm{~d}$ on cube analysis which picture is taken and compared by points to calculate strains occurred and here contours are taken in horizontal and vertical functions of the strength Paregularization parameter which the block sizes of $21 \times 21$ pixels. The DIC method of newton rapshon of adaptive [2]. Measuring the Thermal Expansion Coefficient of Tubular Steel Specimens with Digital Image Correlation Techniques shows weather is it possible to measure the coefficient of thermal expansion (CTE) in steel of DIC technique. Here steels are about (S235 and SS304) and the temperature is about $120 \mathrm{oC}$. Although the strains can occur at small specimens it gives good accurate values by the DIC method. The residual stresses in tubes can come through the welding process of the finite element method by temperature change. The control process of the correlation method shows no influence results. The larger area has some range of values and the smaller range has better than larger occupied values. In input of finite element method, the vague values are suggested and thermal welding technique in cycles, etc are measured [3].Developing Dynamic Digital Image Correlation Technique to Monitor Structural Damage of Old Buildings under External Excitation is about the building capacity to resist the external excitation for the structural design of buildings this research method is using the conventional method here the inter-story drift mode shape based on a characteristic of high sensitivity story drift than approximate story damage index (ADSI) on modal frequency and Vibration which determines a location of buildings damages [4]. Noncontact and Targetless Measurement of Vertical Deflection of Bridges using Off-axis Digital Image Correlation has given solution to get accurate calibration technique by advanced video deflectometer which can track through videography to pixels by converting in image method. In this Gauss-Newton algorithm, a method is implemented. The principle of using a video deflectometer is to get deflection in bridges after that laboratory test is done. Improving stability and robustness is present in the video deflectometer [5]. Digital Image Correlation (DIC) on Fresh Cement Mortor to Quantify Settlement and Shrinkage in this study the water evaporation in cement and cement hydration is very high. Due to plastic shrinkage, the particles are deformed variously and the water bleed has occurred. Here the Digital Image Method is selected to quantify shrinkage strain which is compared to LVDT point in $3 \mathrm{D}$ view deformations the procedure is in the DIC speckle method [6]. Investigation of Transverse Residual Stresses in a Thick Pultruded Composite using Digital Image Correlation with Hole Drilling which detects the matrix cracking before pultrusion in a fiber-reinforced polymer. Here the hole drilling method is to measure the work of transverse strain when the removal of material of thick composite pultruded profile $(20 \times 20)$ made of glass or polyester. This can also be measured in the finite element method which can strain are leveled up and the core profile of transverse stress is found. The hole depth can be 2,3 and $4 \mathrm{~mm}$ in ordered to get reliable data. 
the specimens are prepared to make stress-free by heating them. The finite element gave the complete result of stress-strain during hole drilling. The results are highly accurate in the experiment of error DIC investigation [11]. When the removal of material of thick composite pultruded profile $(20 \times 20)$ made of glass or polyester. This can also be measured in the finite element method which can strain are leveled up and the core profile of transverse stress is found. The hole depth can be 2,3 and $4 \mathrm{~mm}$ in ordered to get reliable data. the specimens are prepared to make stress-free by heating them. The finite element gave the complete result of stress-strain during hole drilling. The results are highly accurate in the experiment of error DIC investigation [12]. Measurement of Coefficient of Thermal Expansion of Films using Digital Image Correlation Method is about the applications in digital image correlation for determination in a coefficient of thermal expansion in the films. There is a heating chamber designed for the application of thermal loads and also provides deformation strain fields. In this $\mathrm{PI} / \mathrm{SiO} 2$ composite film is considered and if lower CTE is taken it gives decreased value so accurate adding filler is more important. Here for strains, $\mathrm{x}$ and $\mathrm{y}$ values are taken from CTE and small rigid body rotation is measured and pure value is considered in films by thermal expansion. The CTE of polyimide composite film temperature range is $20-140 \mathrm{oC}$ is taken. The effective full-field of thermal deformation through CTE is measured. In this high-resolution SEM or optical microscope is considered [13]. Residual Stress in Thermal Spray Coatings Measured by Curvature based on 3D Digital Image Correlation Technique research tells about residual stress in thermally sprayed deposits can affect both process and performance is a very important factor. Here high stress can show the deflection of the structural integrity of sprayed parts and impair the function so it is important to study the stress state by understanding it can be controlled. Here the thermal coatings are given as NiCrCoAIY bonding layer and yttria-stabilized zirconia (YSZ) layer on 304 stainless steel by using three-dimensional digital image correlation as a non-destructive full-field optical measurement method. The stress profile can be analyzed at each and every step by the finite element method based on inverse analysis and stress interpretation of curvature. The stress is analyzed from temperature evolution during post-deposition cooling. By multilayer progressing deposition and elastic-plastic model predictions in models [14]. Thermal Interaction of a Circular Plate-ring structure using Digital Image Correlation Technique and Infrared Heating System tells about the high-speed structures of vehicles which shows the thermal behavior and interactions in high temperature up to $550 \mathrm{oC}$ using noncontact. Here the techniques developed in radiation heating facility, non-contact displacement, and strain measurements [15].
Contact-free Thermal Expansion Measurement of Very Soft Elastomers using Digital Image Correlation which represents the stability of thermal loading for soft elastomers gives the accurate measurements through the apparatus by (CTE) coefficient of thermal expansion here we take a different number of specimens by contact-free measurement method is done. Here the mixing ratio of the separate specimen is 5:1 to 50:1 and CTE of silicone elastomers are measured at high precision. For polydimethylsiloxane (PDMS) of curing temperature and fractional free volume (FFV) is investigated. the proposed method satisfies the accurate CTE of soft materials [16]. aluminum plate specimen is measured during a tensile test by DIC method where it can observe the stresses and strains occurrence. A local strain is more than the local stress and the relationship between both stress and strain are true variations. The stress is derived from the true strain which is an increase of true strain has work hardening. The result of steel is found by maximum strain gets stress\&strain relation precisely. The proof of stress on the tensile test is about the A 1050 plastic strain that starts appearing on the plate. This is based on conventional average strain which works softening but work hardening has occurred and then the value of stress-strain is maximum [17]. Ti foams are materials which are categorized the high surface which is interrelated porosity of high potential for biomedical applications different size, shape, and space are observed and strains of porous materials are considered here the porosity foams are produced by irregular space or any other cubic shape were found for lower yield stress than spherical space holder [18]. The mechanism governing fatigue crack shielding and calculating the stress cracks during crack propagation. This experiment is based on direct and indirect measurement of loads between the crack surface of crack starts and closing. Here the two modern methods established Thermoelastic Stress Analysis (TSA) and 2D Digital Image Correlation Method (2D-DIC) have been conducted tests on 2 aluminum alloys (A12024-T3 and A17050) in different ratios. Results of both tests are quite similar so the stress intensity factor range changes from 0.1 to 0.5 and the potential ability of both methods is for shielding effect due to crack closure [19]. The challenging environmental problems are done through the scheimpflug camera to fill the 3D deformation based on stereo-DIC. This camera can capture without zooming which has a high range. It consists of sensors with a lens for a larger depth field. It has different strategies to calculate intrinsic and extrinsic parameters. The experiment catches the motion and bending test which determines effectiveness and accuracy [21].

2.2 Study of DIC based on mechanical applications PMMA which been treated with guiding tensile knowledge of molecular cable structures which are in 
changed directions and motorized properties depends on particular cable reactions of cracks and tested with various samples which are fractured by considering different loadings here they did the DIC method which can calculate the directions and can be found which approves reliability of molecular cable model. It has lightweight, high-temperature resistance, high light transmittance \& good motorized properties [7]. Digital Image Correlation has mechanical which can withstand the high load, they can present strains due to loadings. DIC method is using one laser beam and the speckle method is created to get mechanical behavior on fiber-reinforced beam. The fibers are kept parallel to the load and the other fibers are kept randomly. We obtain a sensitivity of 1.05 $\times 10-4$ and here $95 \%$ of accuracy has occurred. Strain and stress evolution is observed during tests and good results are observed by comparing in a universal testing machine. Here the standard results are also observed by compression tests [8]. The individual of high pulverized stress in deep ground distresses breakage of rock blasting and adding of high-speed photography technology the digital image correlation method (DIC) it shows about the elastic vibration area then the high stresses range differs from the plastic area or quasielastic area. Here combining a load of high static pressure \& dynamic blasting in stress conditions is studied [9]. Information about rolling direction $(\mathrm{RD}) \&$ the transverse direction (TD) AM50 Mg plate compound where the dic method is applied to get the tensile test, strains has occurred. Here the changes are detected by scanning electron microscope to get the crack types here the critical and simple failures can be detected and the morphology shows RD sample fracture and $\mathrm{Td}$ sample fracture on Alloy.[28]

\subsection{Study of DIC based on Aerospace}

Modeling, Manufacturing \& Testing which gives information on the aerospace applications with has a variety of temperature instigated by varying in environmental situations. The thermal strains cause failure in structures and components for that the aerospace applications are applied. there are types of lattice material with high nodal connectivity the manufacturing can be in $3 \mathrm{D}$ printing and then scales are considered with the DIC method by aerospace applications [22].

2.4 Study of DIC based on biology and food materials

3D DIC based on air plasma spray (APS) coverings layer gives measurement from 22 to $800 \mathrm{oC}$ develops curvature and stresses first we apply zirconia as topcoat stress temperature machinery used to expose the skin. This technique gives and develops the coatings and thin films of dissimilar materials. Appropriate camera and lighting are given by this the curvature, creep is captured by both stress and temperature [20]. Packaging among materials is difficult to measure the mechanical properties due to their softness so to get through the difficulty dic method is applied on the optical method here the flexibility, vibrations of a package all are observed by stress \& strain relationship by poison ratio \& coefficient of heat extension and creep distortion by DIC method so we can get the analysis of measuring range and their stability[27]

\subsection{Study of DIC based on mining}

DIP method for figure properties and relaxing velocity in soil particles from diameter 1 to $10 \mathrm{~mm}$ are taken and tested for a sample. The size of an atom is measured through Vernier calipers and the camera is taken which is Canon EOS100d. the velocity of soil is expressed in the mean diameter \& aspect ratio. Non-spherical soil is taken as and based on it the digital processing is evaluated. The prediction of a formula by the shape, size, and velocity is taken accurately [24].

2.6 Study of DIC based on Nuclear applications Introducing for gauging crack allowance in brittle resources which chains constructions in hightemperature gas-cooled reactor plays a major role in the safety of the reactor. They can afford stations for fuel essentials and coolant streams. DIC method is used to measure the crack extension at the breakage point. The bulk crack curve is important to analyze the R-curve \& tension smoothening behaviors and to know all the stresses or tensions in nuclear graphite crack extension the results are taken by using the finite element method [29].

\subsection{Study of DIC based on meteorology}

True stress-strain is considered by using 3D digital image method conducted at corner areas of cold molded channels which expands ultimate tensile strength and optical trilinear model of two-stage hardening of strain which authenticate reliability optic dimension [10].

2.8 Study of DIC based on traffic signals

Digital Image Correlation: Displacement Accuracy Estimation which gives the information about the accuracy of the techniques more specifically. The image correlation is done by software to get the images and results in accurate and graphical representation is shown. Here the speckle method is more used which gives a good range of noise and size of stresses and deflection occurred in any kind of structures. The function can ensure without any errors and here the French community uses the DIC techniques in such a way to generate the characteristics of the parameters. There are two types of families in the DIC method the very $1 \mathrm{st}$ is about the speckle method and the 2nd one is amplitude error calculation. Through the DIC method, the high noisy images are more efficient and compared to both the families 1st family gives accurate results which is the DIC method [30]. Digital Correlation of Strain Resolution Calculated from In-situ Experiments gives information on SEM imaging to know about strain occurrence in the sample taken by scanning a copy which compares to before and after loading. Here it determines how it varies in shape, size, etc. 
the effect of strain resolution on the DIC method using SEM imaging can exhibit particular signal noise and loss due to inadequate correlation. To know the changes of directions this method is useful to get edge alignment with pointing the directions of strains that occurred and all techniques are on high temperatures on superalloys. The image is about sizes of pixels per speckle the minimum and maximum speckles are sorted in different grid methods and later on it get image frame which is also plotted in graphical representation [31]. According to the above survey we can consider Non-contact digital image correlation method is done on different kinds of materials and these are in different types of departments consists of mechanical, civil, thermal, biological, etc. This method gives accurate output in graphical and diagrammatical representation which is easy in understanding and time-saving.

\section{Future Scope}

1. To get knowledge on Thermal strains of Noncontact digital image correlation method which is economical and gives accurate values.

2. There is high scope to study about thermal strain behavior of steel alloy (railway tracks) from the DIC method.

3. The study of thermal deflection on DIC method can be studied in detail by New techniques and methods can be implemented in Matlab by giving various codes and programs which gives us a graphical representation of strains, we get solutions accurately more in Matlab than compared to manual design.

\section{Conclusion}

- Non-contact DIC is economical and it is a kind of Non- destructive method.

- By using this method we can efficiently measure the strains at minute levels.

- DIC method can be used to get accurate results and program according to it.

- Can easily understand the output by getting graphical representation.

\section{References}

1. Sikarwar, Samiksha, Shakti Singh, and Bal Chandra Yadav. Photonic Sensors 7.4 (2017): 294-304.

2. Cofaru, Corneliu, Wilfried Philips, and Wim Van Paepegem. Applied Optics 49.33 (2010): 6472-6484.

3. De Strycker, Maarten, et al. Optics and Lasers in Engineering 48.10 (2010): 978986.

4. Shih, Ming-Hsiang, and Wen-Pei Sung. Shock and Vibration 2014 (2014).

5. Pan, Bing, Long Tian, and Xiaolin Song. Ndt \& E International 79 (2016): 73-80. 